\title{
Predicting amplitude of solar cycle 24 based on a new precursor method
}

\author{
A. Yoshida and H. Yamagishi \\ National Institute of Polar Research, 10-3, Midoricho, Tachikawa, Tokyo, 190-8518, Japan \\ Received: 8 October 2009 - Revised: 26 December 2009 - Accepted: 29 January 2010 - Published: 8 February 2010
}

\begin{abstract}
It is shown that the monthly smoothed sunspot number (SSN) or its rate of decrease during the final years of a solar cycle is correlated with the amplitude of the succeeding cycle. Based on this relationship, the amplitude of solar cycle 24 is predicted to be $84.5 \pm 23.9$, assuming that the monthly smoothed SSN reached its minimum in December 2008. It is further shown that the monthly SSN in the three-year period from 2006 through 2008 is well correlated with the monthly average of the intensity of the interplanetary magnetic field (IMF). This correlation indicates that the SSN in the final years of a solar cycle is a good proxy for the IMF, which is understood to reflect the magnetic field in the corona of the sun, and the IMF is expected to be smallest at the solar minimum. We believe that this finding illuminates a physical meaning underlying the well-known precursor method for forecasting the amplitude of the next solar cycle using the $a a$ index at the solar minimum or its average value in the decaying phase of the solar cycle (e.g. Ohl, 1966), since it is known that the geomagnetic disturbance depends strongly on the intensity of the IMF. That is, the old empirical method is considered to be based on the fact that the intensity of the coronal magnetic field decreases in the late phase of a solar cycle in parallel with the SSN. It seems that the precursor method proposed by Schatten et al. (1978) and Svalgaard et al. (2005), which uses the intensity of the polar magnetic field of the sun several years before a solar minimum, is also based on the same physical phenomenon, but seen from a different angle.
\end{abstract}

Keywords. Solar physics, astrophysics, and astronomy (General or miscellaneous)

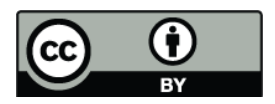

Correspondence to: A. Yoshida (akio.yoshi@nifty.com)

\section{Introduction}

Many estimates concerning the size of the approaching solar cycle have been performed using different methods. A summary and analysis of more than 50 predictions for the amplitude of cycle 24 is presented in Pesnel (2008). The range of the estimated amplitude values is surprisingly wide. Some predict that the maximum sunspot number for cycle 24 will be as large as 180 (Dikpati et al., 2006), while others estimate that it will be as small as 75, making the approaching solar cycle the smallest in the last 100 years (Svalgaard et al., 2005). High solar activity is likely to be associated with numerous large eruptions on the sun's surface, which generate high-energy particles and severe geomagnetic storms that affect the orbital motion of satellites, operations of astronauts in space, communication systems, and electric power lines. Consequently, knowing precisely the level of future solar activity is an important issue for human activities and societies. Empirical methods try to predict the size of the approaching cycle based on the sunspot number or geomagnetic activity at the solar minimum. However, most previous predictions were performed based on the data collected before 2005 or 2006, at which point the solar activity had not yet reached its minimum. Therefore, it is expected that the amplitude of the approaching solar cycle can be more accurately predicted at present.

In the present paper, a new precursor method for predicting the magnitude of the forthcoming solar activity is proposed. The method is based on our finding that the rate of decrease of the SSN or the monthly smoothed SSN in the late years of the solar cycle is correlated with the amplitude of the succeeding cycle. A tendency has been pointed out that the more rapidly the SSN increases toward a maximum, the larger the amplitude of that cycle (e.g., Thompson, 1988), but it has not been recognized that the faster the decay of the SSN towards a minimum, the larger the amplitude of the succeeding cycle. The critically important point is not the average rate

Published by Copernicus Publications on behalf of the European Geosciences Union. 
(a)

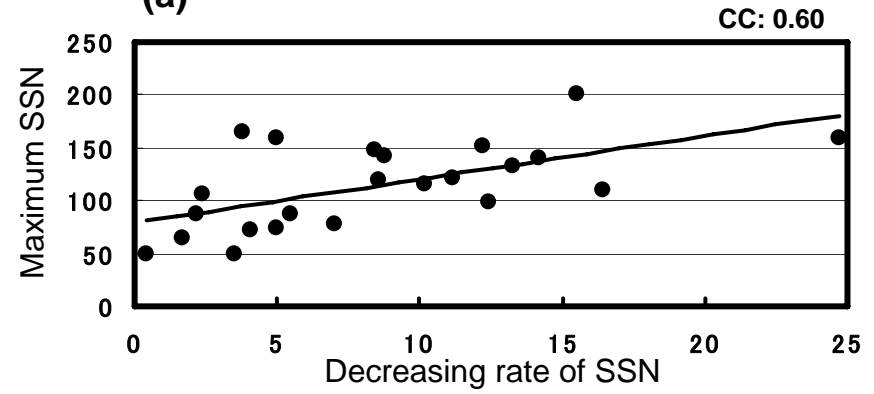

(b)

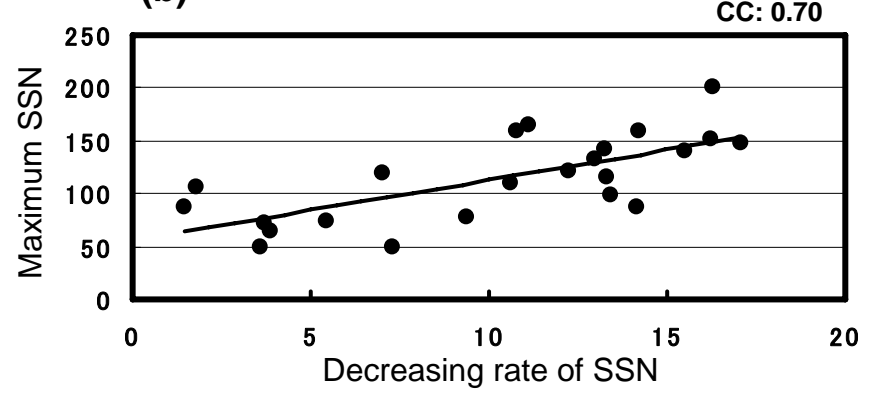

(c)

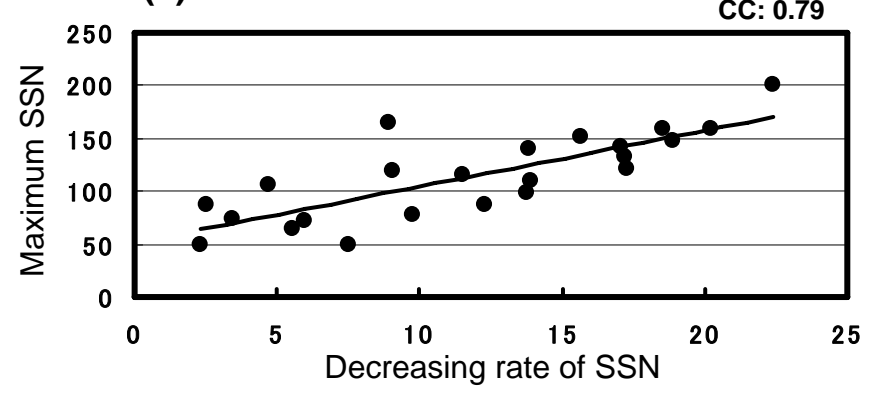

(d)

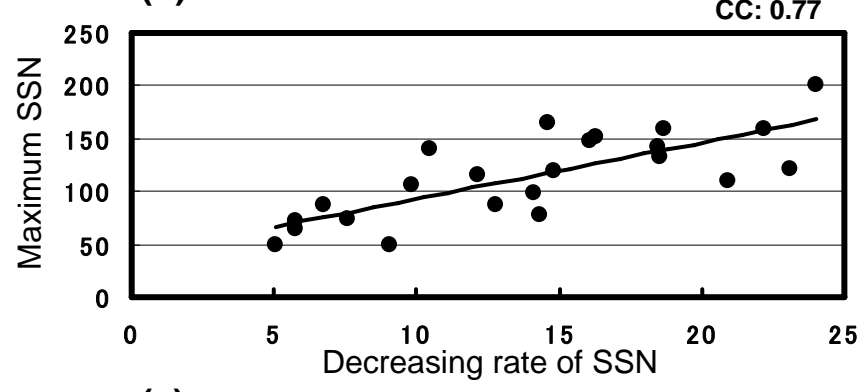

(e)

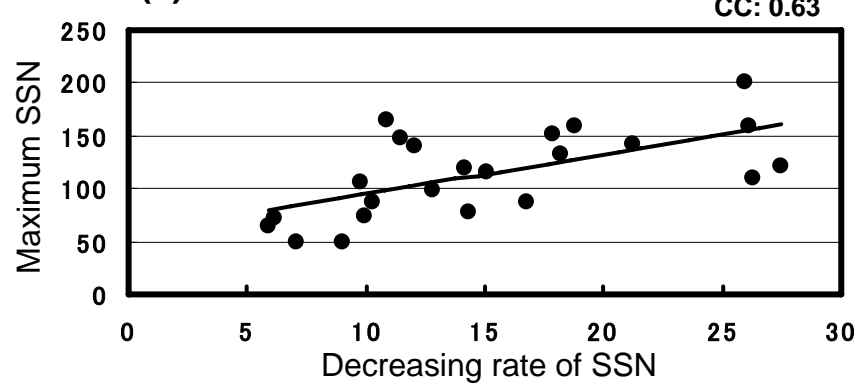

(f)

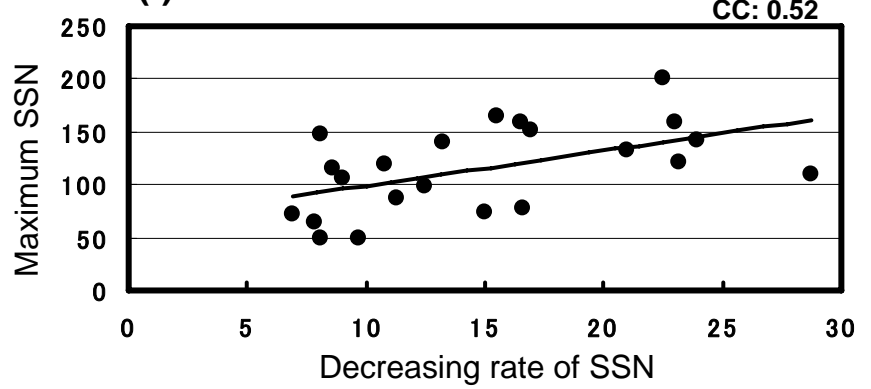

Fig. 1. (a) Peak sunspot number versus rate of decrease in the preceding cycle over a one-year period before the minimum. Panels (b), (c), (d), (e) and (f) are the same as panel (a) but for two-year, three-year, four-year, five-year and six-year periods before the minimum, respectively.

of decrease of the SSN during the entire period of the decay phase, but instead that during the several years before the minimum or, alternatively, the average monthly SSN during the late years of a solar cycle.

It was reported recently that the length of a solar cycle period is correlated with the amplitude of the next solar cycle (Watari, 2008). However, it was a wonder that no correlation was found between the lengths of either the rising or decaying phases of the cycle and the size of the succeeding cycle (Watari, 2008). This paper provides the answer to this puzzle by demonstrating that the significant parameter which is related to the size of the next cycle is not the total length of a cycle or the length of its decay period, but rather the length of the final part of the cycle, after the SSN has fallen to a value of 50 or so.

We further show that the monthly average SSN in the late stage of a solar cycle is a good proxy for the intensity of the interplanetary magnetic field (IMF), and discuss the implications of this observation. The correlation between the SSN and the IMF is considered to provide the physical background for the well-known precursor method to forecast the size of a succeeding solar cycle based on the annual or monthly minimum value of the index for global geomagnetic disturbance (Ohl, 1966).

\section{A newly found precursor}

Figure 1a through $\mathrm{f}$ shows scatter plots of the peak of the smoothed SSN in the succeeding cycle with respect to the rate of decrease in the number of sunspots over the course of one, two, three, four, five and six years, respectively, before the minimum. Data related to the monthly SSN was obtained from the Solar Influence Data Analysis Center (http://www. sidc.be/sunspot-data/). It is seen that the peak of the SSN is correlated with the rate of decrease over all six periods, but the best correlation is obtained for the rate of decrease over 


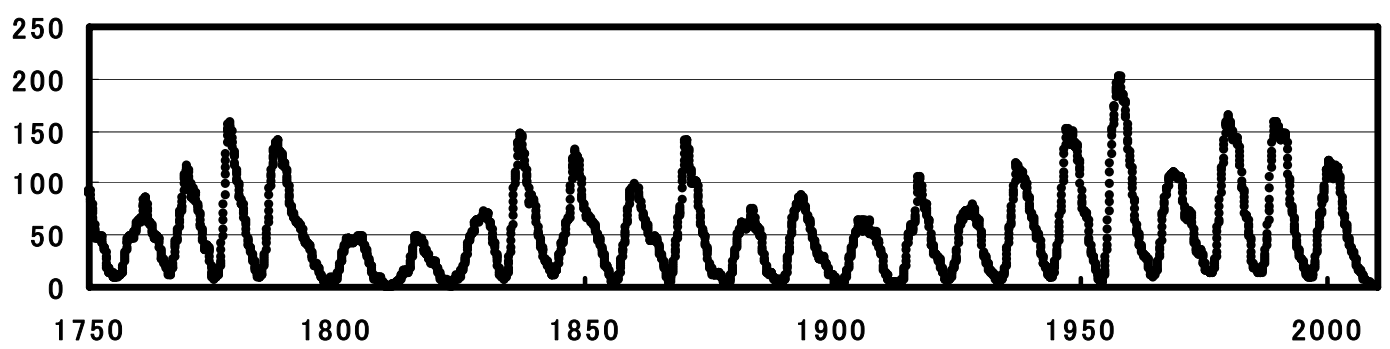

Fig. 2. Monthly smoothed sunspot number since 1750 .

(a)

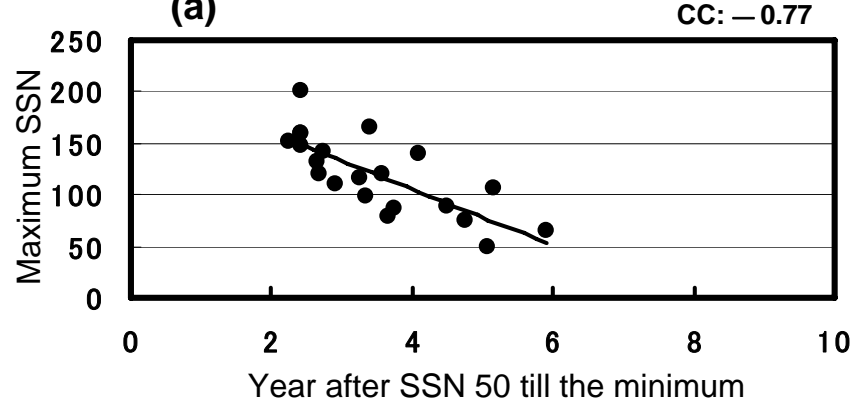

(b)

CC: 0.26

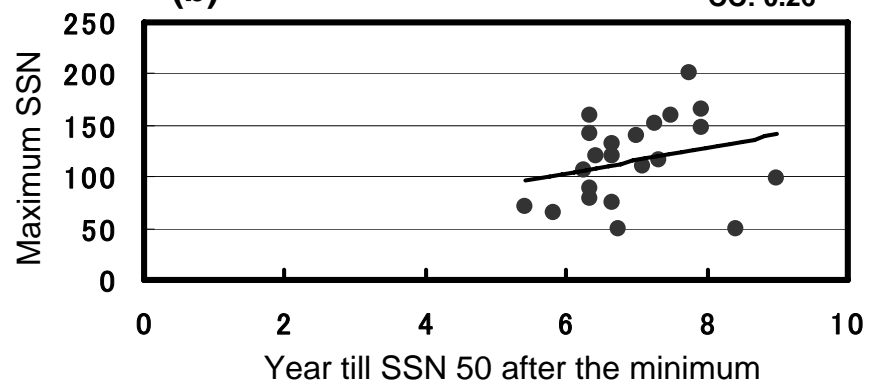

Fig. 3. (a) Peak sunspot number in the succeeding cycle versus time required for the sunspot number to decrease from a value of 50 to its minimum value during the decaying phase. (b) Peak sunspot number in the succeeding cycle versus time required for the sunspot number to increase from its minimum, pass through its maximum, and decrease to a value of 50 .

the course of three years before the minimum. On the other hand, Thompson (1988) showed that the rate of increase of the SSN after the minimum is correlated with the amplitude of that cycle. It is interesting to note that, in that case also, the best correlation was obtained with the rate of increase calculated over a three-year period.

Our findings and those by Thompson (1988) seem not to accord with the results recently reported by Watari (2008), who found no correlation between the length of either the increasing or decreasing phase on the maximum SSN in the succeeding cycle. However, this apparent contradiction can be resolved if we note that what is actually relevant to the amplitude of the next cycle is not the length of the increasing or decreasing phases, but the rate of increase or decrease. A more intriguing result presented by Watari (2008), which was also shown by Hathaway et al. (2002), is that the length of the solar cycle is negatively correlated with the maximum SSN of the succeeding cycle. Why does the sum of the lengths of the rising and decaying phases show a significant negative correlation with the amplitude of the next solar cycle, when the individual lengths of each phase do not? We found that this can also be resolved by considering the fact that a higher rate of decrease implies a shorter time period between some prescribed value of the SSN and the minimum value, and not necessarily a shorter period for the whole decay phase.

While looking at a graph depicting the monthly smoothed SSN since 1750 (Fig. 2), we noticed a tendency that the shorter the interval below a certain value of the SSN, the larger the succeeding peak of the SSN. Figure $3 a$ and $b$ shows scatter plots of the maximum smoothed SSN in the succeeding cycle with respect to the time required for the SSN to fall from a value of 50 to its minimum value, and with respect to the time required for the $\mathrm{SSN}$ to rise from its minimum value, reach a peak and then fall to a value of 50, respectively. It is obvious that a good negative correlation is observed in the former plot, but not in the latter plot. This clearly indicates that the really significant parameter related to the amplitude of the succeeding solar cycle is not the entire length of the preceding solar cycle, but the length of the late stage of the decaying phase of the cycle. As an aside, the average SSN at times 3 and 4 years before the minimum is 41 and 61, respectively, so that a SSN value of 50 corresponds to a time somewhere between 3 and 4 years before the minimum for each cycle.

When the smoothed monthly SSN itself is used instead of the rate of decrease, a similar correlation is also observed. Figure 4a through $\mathrm{f}$ shows scatter plots of the peak of the smoothed SSN in the succeeding cycle with respect to the smoothed SSN at a time, one, two, three, four, five and six years before the minimum, respectively. Here, it should be noted that the smoothed SSN for any given month is the average of the monthly SSN values for a 12-month period, centered on that month (http://www.sidc.be/sunspot-data/). From Fig. 5, where the correlation coefficients for the cases 
(a)

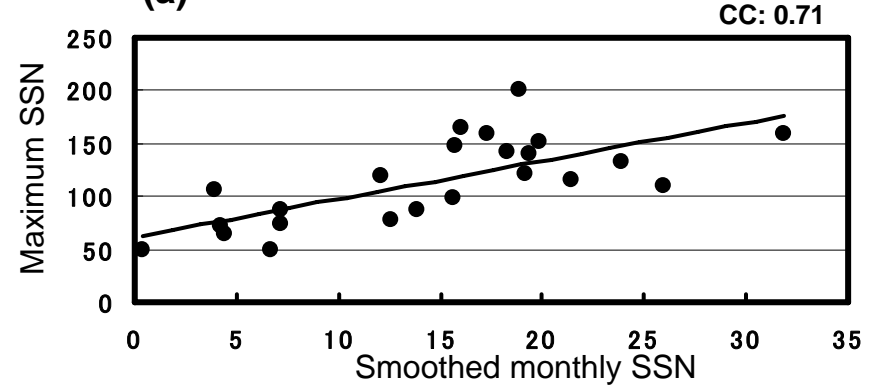

(b)

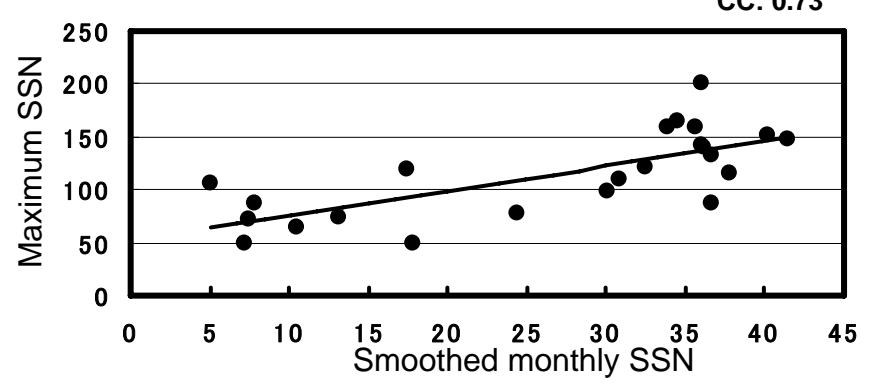

(c)

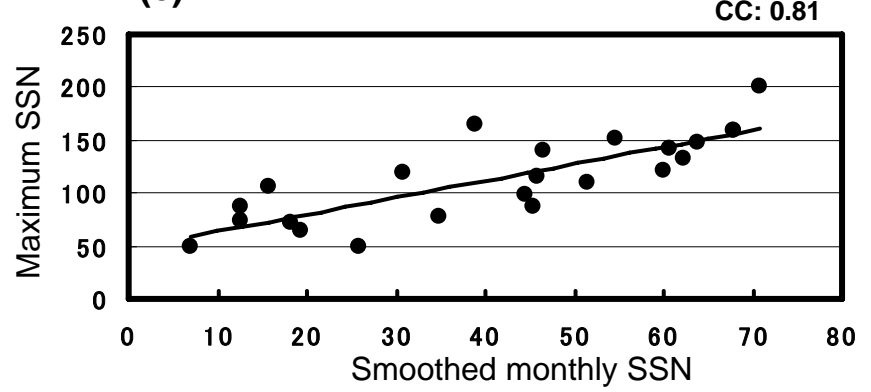

(d)

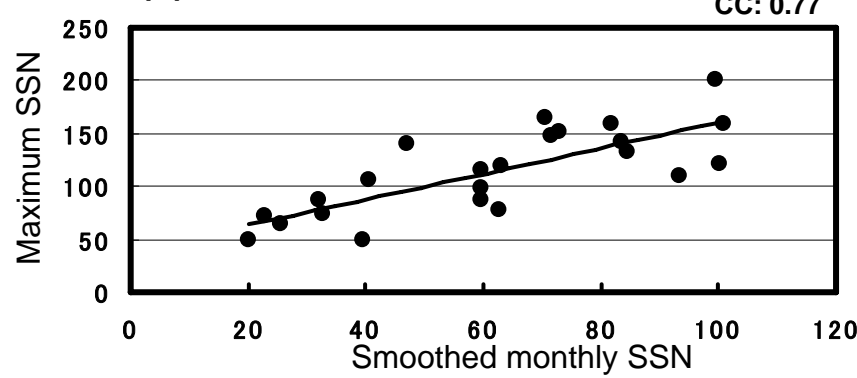

(e)

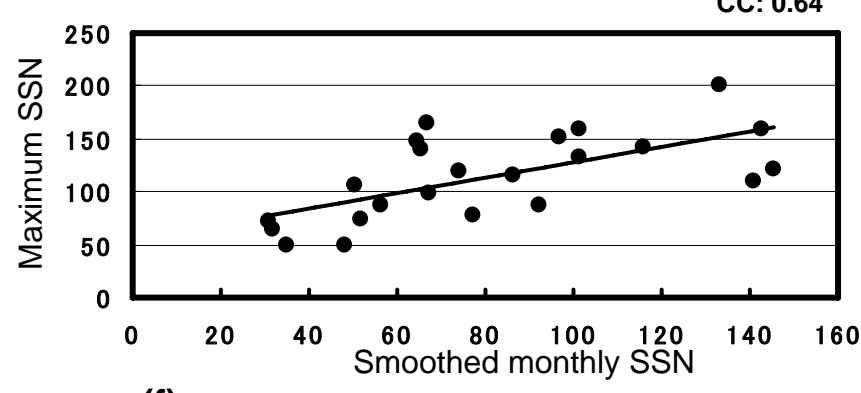

(f)

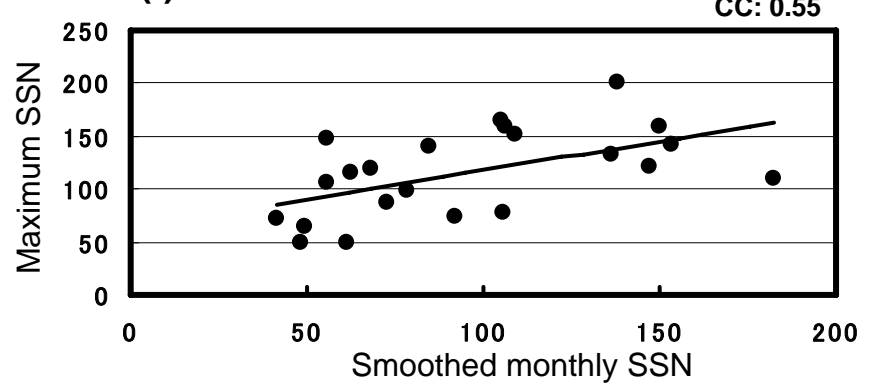

Fig. 4. (a) Peak sunspot number in the succeeding cycle versus monthly smoothed sunspot number one year before the minimum. Panels (b), (c), (d), (e) and (f) are the same as panel (a) but for two-year, three-year, four-year, five-year and six-year periods before the minimum, respectively.

shown in Fig. 1 and in Fig. 4 are compared, it is seen that the coefficients for both data sets agree very well except for the case of one year before the minimum, where a higher correlation is found between the succeeding-cycle amplitude and the monthly smoothed SSN. The data point at 0 year in Fig. 5 represents the correlation between the minimum SSN and the following maximum SSN, where the rate of decrease of the SSN is supposedly zero so that the corresponding correlation coefficient does not exist. The general agreement of the correlation coefficients for the two data sets is not surprising, if we note that the rate of decrease for any given month is calculated as (monthly smoothed SSN at that point - minimum SSN)/(interval between the time in question and the time when the SSN has a minimum value). Therefore, if the monthly smoothed SSN at the time in question is sufficiently larger than the minimum SSN, the rate of decrease will become almost proportional to the monthly smoothed SSN value. In fact, very good correlation is observed be- tween the monthly smoothed SSN and its rate of decrease for 4 or more years before the minimum, as is shown in Fig. 6 .

We believe, however, that it is significant that the highest correlation between the monthly smoothed SSN and the amplitude of the succeeding solar cycle is obtained at a time 3 years before the minimum, as will be discussed later.

\section{Prediction of the amplitude of solar cycle 24}

As described in the preceding section, the monthly smoothed SSN several years before the minimum can be regarded as a precursor indicating the amplitude of the coming solar cycle. If the monthly smoothed SSN is assumed to have taken a minimum value in December 2008, the amplitude of solar cycle 24 is predicted to be $84.5 \pm 23.9$ based on the correlation between the monthly smoothed SSN 3 years before the minimum and the maximum monthly SSN of the succeeding cycle. The red circle in Fig. 7 shows the predicted value, 


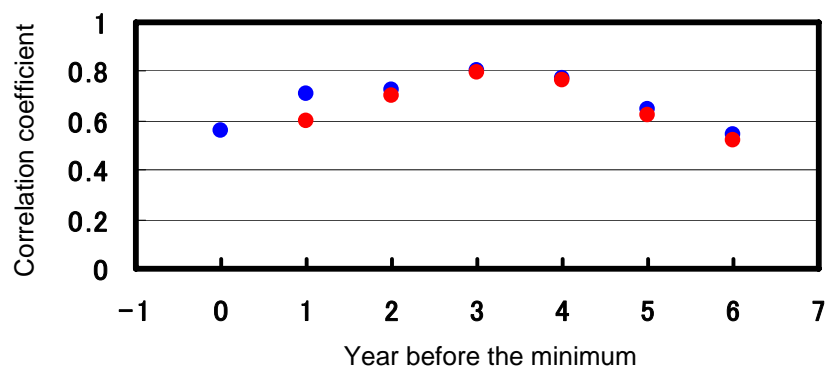

Fig. 5. Blue circles: Correlation coefficient between the maximum sunspot number in the succeeding cycle and the monthly smoothed sunspot number at the minimum and at one, two, three, four, five, and six years before the minimum. Red circles: Correlation coefficient between the maximum sunspot number in the succeeding cycle and the average rate of decrease of the monthly smoothed sunspot number over a time period of one, two, three, four, five, and six years before the minimum.

which is smaller than the value of $101.1 \pm 15.9$ predicted by Watari (2008) based on the relationship between the length of a cycle and the maximum sunspot number of the next cycle.

Dikpati et al. (2006) predicted that cycle 24 will have a 30$50 \%$ larger amplitude than cycle 23 , indicating that the peak sunspot number will be as large as 155-180. Their method is based on a flux-transport dynamo model, which states that the polar fields become advected to the shear layer at sunspot latitudes after 17-21 years, depending on the meridional flow strength (Dikpati et al., 2004). According to the model, the polar fields from the past few cycles $(n-1, n-2, n-3)$, rather than just from the declining phase of the previous cycle $(n-1)$ as supposed by Schatten et al. (1978), influence the spot-producing toroidal field strength of cycle n. However, only one observational fact is known which shows the prolonged relationship between solar cycles. Hathaway et al. $(2003,2004)$ reported that the drift velocity of the centroid of the sunspot area toward the equator at the sunspot cycle maximum in cycle $n$ is related to the amplitude of cycle $(n+2)$. However, they also pointed out that the correlation only explains $50 \%$ of the variance in cycle amplitude (Hathaway et al., 2004). No indication of a relationship between cycle $(n-2)$ and cycle $\mathrm{n}$ has been found in the analysis of SSN or geomagnetic indices. In addition, we could not find any relationship between the monthly smoothed SSN in the decaying phase of cycle $\mathrm{n}$ and the amplitude of cycle $(n+2)$.

In the dynamo model developed by Babcock (1961) and Leighton (1969), the polar field at solar minimum is considered to serve as a "seed" for the toroidal field that comes to the surface in the succeeding cycle to form active regions. Assuming that the strength of the polar fields during the decay phase, which reach their peak amplitudes several years after the sunspot maximum, is an indicator of peak sunspot activity for the following cycle, Svalgaard et al. (2005) predicted that the amplitude of solar cycle 24 will be the smallest in the last 100 years, with a peak smoothed monthly SSN of $75 \pm 8$. Their method is originally from Schatten et al. (1978). Schatten (2005) performed a similar prediction by reexamining polar field data and using proxies of the sun's polar field, such as soft X-rays from coronal holes and polar faculae, and forecasted fair space weather for cycle 24, meaning the amplitude will not be large, with a smoothed SSN of about $80 \pm 30$.

Another well-known precursor method developed by Ohl and his group $(\mathrm{Ohl}, 1966)$ uses the relationship between the minimum of geomagnetic activity (where the $a a$ index is usually used for this purpose) and the sunspot maximum of the next cycle. Based on the precursor method, Kane (2007) estimated the amplitude of cycle 24 to be $124 \pm 26$, using the $a a$ data collected up to 2006. Based on a similar method, but using Feynman's I-component (Feynman, 1982) of the $a a$ index in the decreasing phase of the preceding cycle, Hathaway and Wilson (2006) estimated the maximum SSN of cycle 24 to be $160 \pm 25$. This value is even larger than that estimated by Kane (2007). Recently, Bhatt et al. (2009) obtained a value of $92.8 \pm 19.6$ for the amplitude of annual mean SSN of cycle 24 using the relationship between the average of $a a$ index for the year of the sunspot minimum and the preceding four years and the amplitude of the succeeding solar cycle regarding 2008 as the minimum year. This prediction is almost in agreement with ours. In the next section we explain the reason why the empirical methods based on the geomagnetic activity tend to give large values.

\section{Discussion}

It seems that different precursor methods are grounded on the same root. Figure 8a, b, c exhibits the change in monthly smoothed SSN, monthly average of the total intensity of the IMF, and the solar wind velocity in the sun-earth direction, respectively, during the period from 1998 through 2008. Note that the IMF began to decrease noticeably starting in the end of 2005. As discussed in Sect. 2, the timing is consistent with the period for which the monthly smoothed SSN or the rate of decrease is well correlated with the amplitude of the next cycle. It is remarkable that the monthly average of the intensity of the IMF is so well correlated with the monthly SSN in the three years from 2006 through 2008, as is shown in Fig. 9a. This indicates that the monthly smoothed SSN in the late years of the decaying phase is a good proxy for the monthly average of the intensity of the IMF, which is thought to reflect the magnetic field in the corona of the sun, and the IMF should be smallest at the solar-cycle minimum. Therefore, we believe that the overall solar magnetic field or the coronal magnetic field is the key factor underlying the precursor methods for predicting the amplitude of the approaching cycle, and in the late stages of solar cycle its intensity is well represented by the SSN. 
(a)

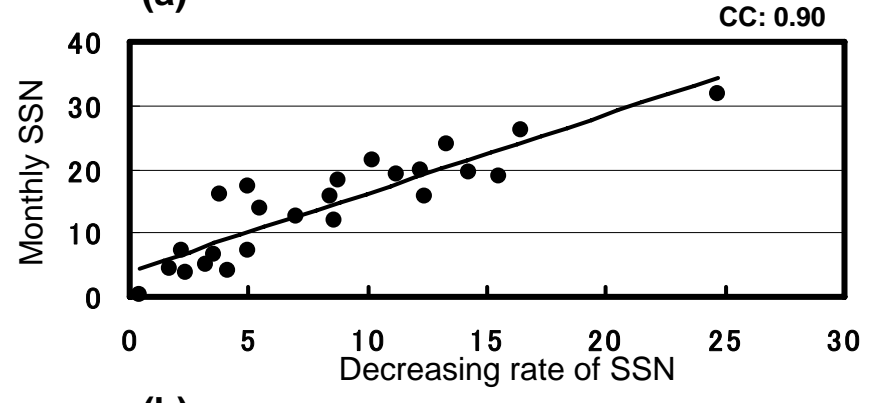

(b)

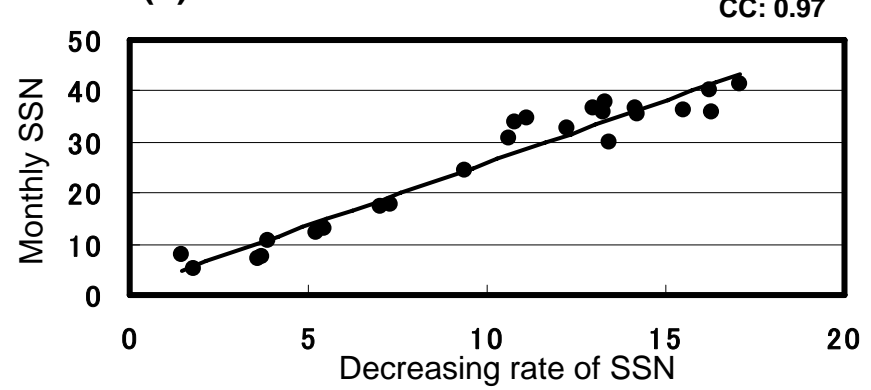

(c)

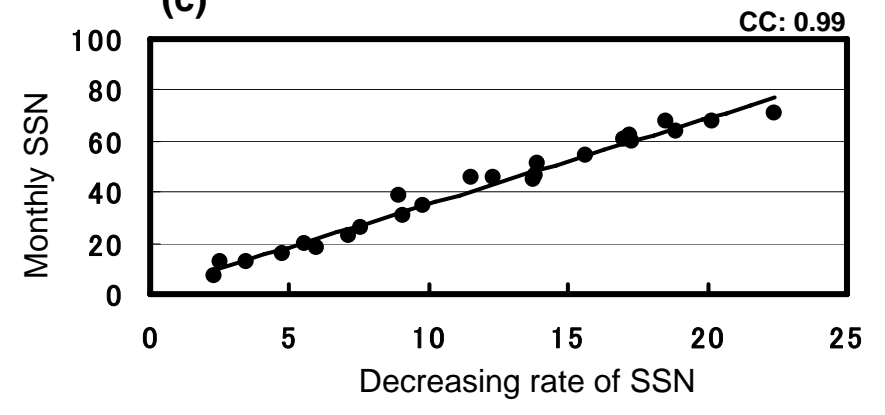

(d)

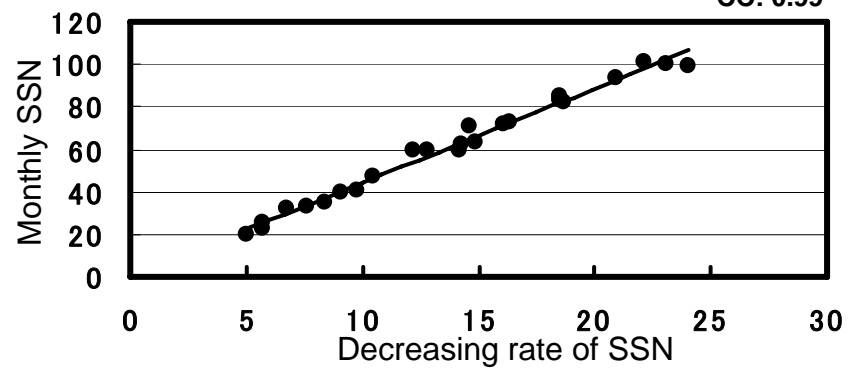

(e)

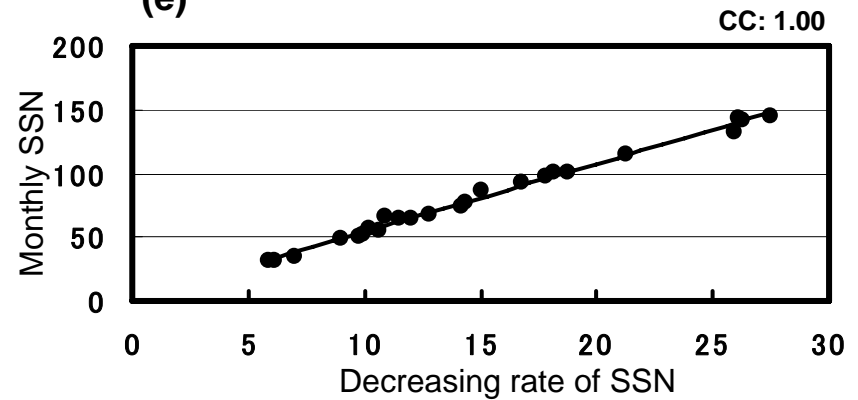

(f)

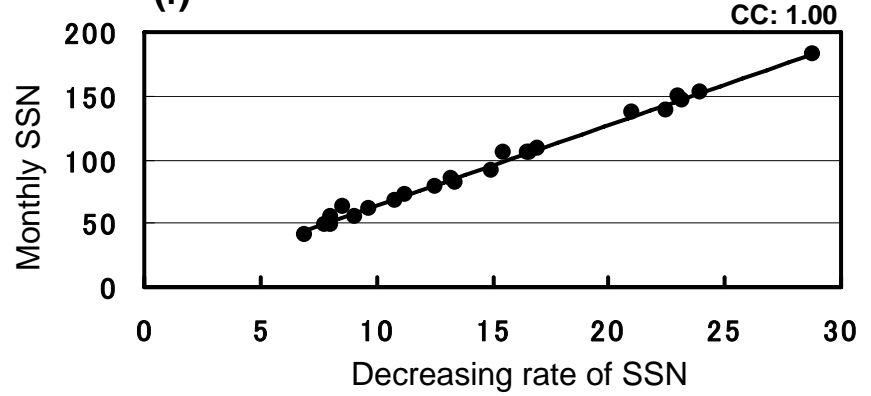

Fig. 6. (a) Monthly smoothed sunspot number one year before the minimum versus average rate of decrease of monthly smoothed sunspot number during a one-year period before the minimum. Panels (b), (c), (d), (e) and (f) are the same as panel (a) but for two-year, three-year, four-year, five-year and six-year periods before the minimum, respectively.

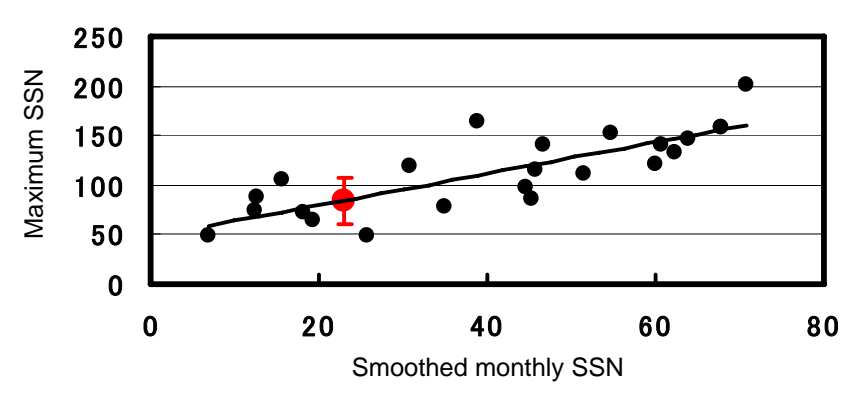

Fig. 7. Scatter plot of maximum sunspot number in the succeeding cycle versus monthly smoothed sunspot number three years before the minimum. The red circle shows the predicted maximum sunspot number for cycle 24 and the bar indicates 1-sigma accuracy.

Although the physical basis of the well-known precursor method using the $a a$ index at the solar-cycle minimum or its average value in the decaying phase of the solar cycle (e.g.
Ohl, 1966) has never been well understood, the above discussion appears to offer some clues, for it is known that the geomagnetic disturbance depends strongly on the intensity of the IMF. In other words, the old empirical method is considered to be grounded on the fact that the intensity of the coronal magnetic field decreases in the final years of the solar cycle in parallel with the SSN. Although not shown here, the rate of decrease of the SSN during the three-year period before the minimum is well correlated with the minimum $a a$ index.

As mentioned before, Hathaway et al. (2006) predicted a large amplitude for the SSN in cycle 24 based on the Feynman I-component of geomagnetic activity in the decreasing phase. Here, it should be noted that mid-latitude indices such as $a a$ are correlated to the product of the square of the solar wind velocity and the southward component of the IMF in GSM coordinates (e.g., Maezawa and Murakami, 1986; Yoshida, 2009). Although the IMF has begun to decrease 
(a)

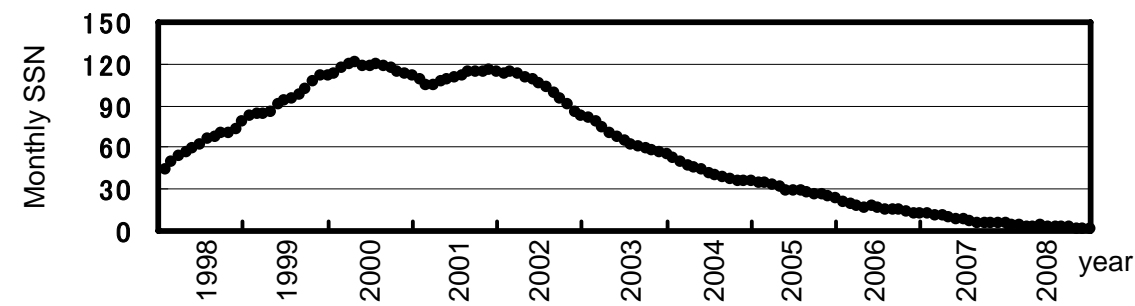

(b)

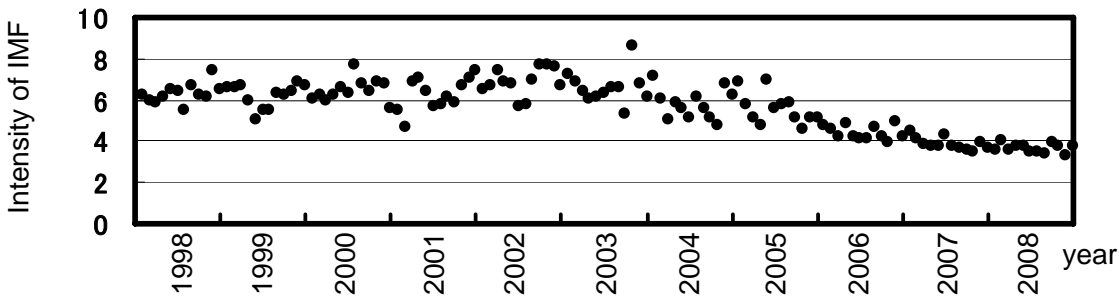

(c)

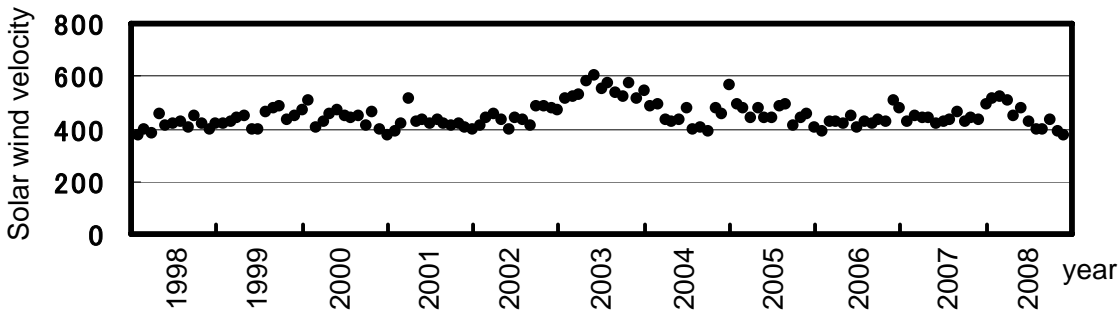

Fig. 8. Change in monthly smoothed sunspot number (a), change in total intensity of the interplanetary magnetic field (b), and change in the solar wind in the sun-earth direction during the period from 1998 through 2008 (c). Level 2 hourly data taken by the ACE satellite is used for the interplanetary magnetic field and the solar velocity. The units for the magnetic fields are $\mathrm{nT}$ and solar wind velocity is km/s.

since approximately 2003, the solar wind velocity in 2003 was exceptionally high, as shown in Fig. 8c, which produced an extraordinarily large geomagnetic disturbance (Yoshida, 2008). In the analysis of Hathaway et al., the effect of the high solar wind velocity in 2003 was not taken into consideration, which is the reason for their prediction of a large maximum SSN for cycle 24 . We think it is essential for any precursor method to provide a proper prediction that the method is based on a proxy that reflects the change in the magnetic field of the sun or the IMF. In that regard, the method based on the $a a$ index seems a little problematic in the sense that it does not properly take into account the effects of the solar wind velocity.

The precursor method proposed by Schatten et al. (1978) and Svalgaard et al. (2005), which is based on the polar magnetic field of the sun, or the magnitude of the sun's dipole moment, several years before a solar minimum is also considered to be grounded on the same physical phenomenon, i.e., the overall magnetic field of the sun. It is interesting that Svalgaard et al. (2005) used the average intensity of the polar magnetic field during the final three years of three solar cycles for the prediction of the size of cycle 24 . Why was a value of three years used? We have shown that the monthly smoothed SSN at a time three years before the minimum or its rate of decrease during those three years is best correlated to the amplitude of the succeeding cycle using SSN data for (a)

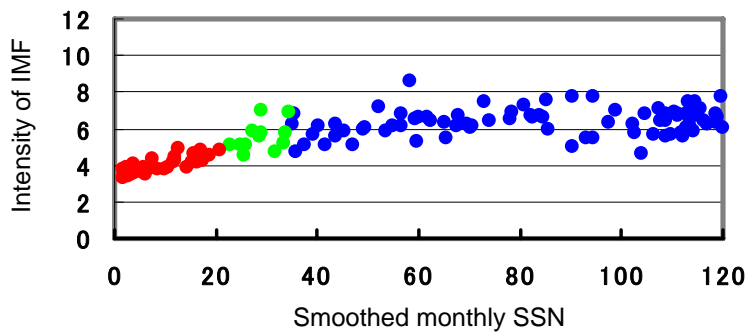

(b)

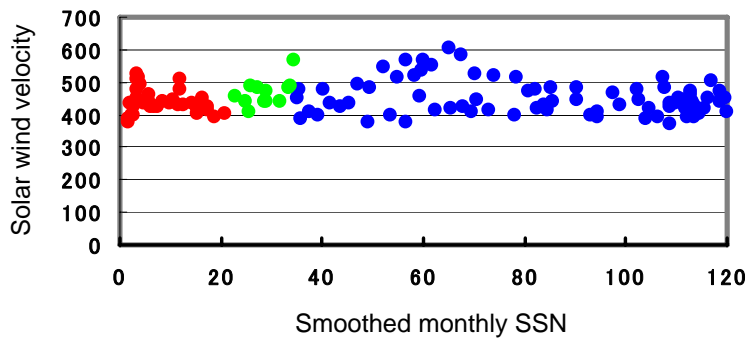

Fig. 9. (a) Scatter plot between monthly average of the total intensity of the interplanetary magnetic field and the monthly smoothed sunspot number. (b) Scatter plot between monthly average of the solar wind velocity in the sun-earth direction and the monthly smoothed sunspot number. Red, green and blue circles represent data for the years 2006 through 2008, 2005, and 1998 through 2004, respectively. 
23 cycles since 1750 . Here, we point out that the average SSN during the final three years of solar cycles is not so well correlated with the size of the next cycle as the SSN at a time three years before the minimum.

We think it is very probable that the overall magnetic field of the sun in the final years of a solar cycle reflects some phenomenon related to the generation of the solar magnetic field for the next cycle. It appears that this process begins three to four years before the minimum SSN is reached, and not early in the decreasing phase. Cameron and Schussler (2007), having studied the origin of the predictive skill of a kinematic flux-transport model to forecast the amplitude of solar cycle, found that sunspot activity in low latitude during a few years before the solar minimum plays an essential role in the predictability. Their observation is in good agreement with our finding. Our method should be practically most useful if the minimum epoch of the SSN can be forecasted in an early stage of the declining phase. Cameron and Schussler (2007) claim that the epoch of the minimum is determined by the so-called Waldmeier effect and overlapping of cycles, that is, the epoch as well as the minimum value of the SSN depends on the strength of the following cycle: When it is strong, the epoch shifts to the early time and the minimum SSN becomes larger. In that case, it would be difficult to know the minimum epochs beforehand. We think, however, that the appearance of the correlation between the SSN and the IMF three to four years before the solar minimum is suggestive and expect that it affords us a clue to anticipate the minimum epoch before it is reached.

\section{Conclusion}

We predict the amplitude of solar cycle 24 to be $84.5 \pm 23.9$ based on the correlation between the monthly smoothed sunspot number (SSN) at a time three years before the solar minimum and the amplitude of the next cycle, assuming that the monthly smoothed SSN reached its minimum in December 2008. Further, having shown that the monthly SSN in the three years from 2006 through 2008 is well correlated with the monthly average of the intensity of the interplanetary magnetic field (IMF), we suggest that the physical basis of the well-known precursor method for forecasting the amplitude of the next solar cycle using geomagnetic activity is related to the magnitude of the IMF or the coronal magnetic field of the sun in the late stages of a solar cycle, which is thought to be related to the generation of the solar magnetic field of the next cycle. The precursor method based on the intensity of the polar magnetic field of the sun several years before a solar minimum is also considered to be based on the same physical phenomenon.

Acknowledgements. We thank the SIDC-team, World Data Center for the Sunspot Index, Royal Observatory of Belgium for the smoothed monthly mean SSNs, the ACE SWEPAM instrument team and the ACE Science Center for providing the ACE data, and
S. Watari for valuable discussions. Comments by D. Willis and an anonymous referee are gratefully acknowledged.

Topical Editor R. Forsyth thanks D. Willis and another anonymous referee for their help in evaluating this paper.

\section{References}

Babcock, H. W.: The topology of the Sun's magnetic field and the 22-year cycle, Astrophys. J., 133, 572-587, 1961.

Bhatt, N. J., Jain, R., and Aggarwal, M.: Prediction of the maximum amplitude and timing of sunspot cycle 24, Solar Phys., 260, 225232, 2009.

Cameron, R. and Schussler, M.: Solar cycle prediction using precursors and flux transport models, Astrophy. J., 659, 801-811, 2007.

Dikpati, M., de Toma, G., and Gilman, P. A.: Predicting the strength of solar cycle 24 using a flux-transport dynamo-based tool, Geophys. Res. Lett., 23, L05102, doi:10.1029/2005GL025221, 2006.

Dikpati, M., de Toma, G. Gilman, P. A., Arge, C. N., and White, O. R.: Diagnostics of polar field reversal in solar cycle 23 using a flux-transport dynamo model, Astrophys. J., 601, 1136-1151, 2004.

Feynman, J.: Geomagnetic and solar wind cycles, 1900-1975, J. Geophys. Res., 87, 6153-6162, 1982.

Hathaway, D., Nandy, H. D., Wilson, R. M., and Reichmann, E. J.: Evidence that a deep meridional flow sets the sunspot cycle period, Astrophys. J., 589, 665-670, 2003.

Hathaway, D. H., Nandy, D., Wilson, R. M., and Reichmann, E. J.: Erratum: "Evidence that a deep meridional flow sets the sunspot cycle period, Astrophys. J., 602, 543, 2004.

Hathaway, D. H. and Wilson, R. M.: Geomagnetic activity indicates large amplitude for sunspot cycle 24, Geophys. Res. Lett., 33, L18101, doi:10.1029/2006GL027053, 2006.

Hathaway, D. H., Wilson, R. M., and Reichmann, E. J.: Group sunspot numbers: Sunspot cycle characteristics, Solar Phys., 211, 357-370, 2002.

Kane, R. P.: A preliminary estimate of the size of the coming solar cycle 24, based on Ohl's precursor method, Solar Phys., 243, 205-217, 2007.

Leighton, R. B.: A magneto-kinematic model of the solar cycle, Astrophys. J., 156, 1-26, 1969.

Maezawa, K. and Murakami, T.: Solar wind velocity effects on the auroral zone magnetic disturbances, in: Solar WindMagnetosphere Coupling, edited by: Kamide, Y. and Slavin, J. A., pp. 59-83, Terra Scientific, Tokyo, 1986.

Ohl, A. I.: Forecast of sunspot maximum number of cycle 20, Solice Donie, 9, 84-85, 1966.

Pesnell, W. D.: Prediction of solar cycle 24, Solar Phys., 252, 209220, 2008.

Schatten, K. H.: Fair space weather for solar cycle 24, Geophys. Res. Lett., 32, L21106, doi:10.1029/2005GL024363, 2005.

Schatten, K. H., Scherrer, P. H., Svalgaard, L., and Wilcox, J. M.: Using dynamotheory to predict the sunspot number during solar cycle 21, Geophys. Res. Lett., 5, 411-414, 1978.

Svalgaard, L., Cliver, E. W., and Kamide, Y.: Sunspot cycle 24: Smallest cycle in 100 years?, Geophys. Res. Lett., 32, L01104, doi:10.1029/2004GL021664, 2005.

Thompson, R. J.: The rise of solar cycle number 22, Solar Phys., 117, 279-289, 1988. 
Watari, S.: Forecasting solar cycle 24 using the relationship between length and maximum sunspot number, Space Weather, 6, S12003, doi:10.1029/2008SW000397, 2008.

Yoshida, A.: Why was the geomagnetic activity in 2003 extraordinarily high?, Japan GeoScience Union Meeting 2008, E113-011, 2008.
Yoshida, A.: Physical meaning of the equinoctial effect for semiannual variation in geomagnetic activity, Ann. Geophys., 27, 1909-1914, 2009,

http://www.ann-geophys.net/27/1909/2009/. 